\title{
ELECTROMAGNETIC EMERGENCE IN METAMATERIALS
}

\author{
Deconstruction of terminology of complex media
}

\author{
A. SIHVOLA \\ Helsinki University of Technology, Electromagnetics Laboratory \\ P.O. Box 3000, FIN-02015 HUT, Finland
}

\section{Introduction. The power of naming things.}

"Metamaterials." This word has become very common in the recent discussion of materials research in electromagnetics, and perhaps also in some other fields of applied and theoretical physics. At least such an impression is by no means excluded. If this is true, what is the reason for such a state of affairs? Why is "metamaterial" used increasingly in electromagnetics parlance, if not yet so much in literature? Indeed, what is the exact meaning of the term?

In this presentation, I would like to problematise the use of the term "metamaterial," at least in the extent to which it is used exclusively to new, especially nanoengineered, materials. The history of electromagnetics research contains a very rich collection of results for random, inhomogeneous, and composite materials. A closer look at the properties of these old-fashioned materials reveals that they could be certainly classified into the group of the modern metamaterials, even though they may be well known and familiar to many of us as "ordinary" materials.

That scientists name things such that they attract attention is understandable. One can think of the recent surge of interest in PBG (photonic band gap) materials, or photonic crystals, where artificially manufactured geometries create pass band and stop bands for electromagnetic waves in microwave, millimetre wave, and optical regions. Physicist-driven research on PBG's has caused irritation within the microwave engineering community, and engineers point out (partially correctly) that periodical dielectric materials are nothing new. But who would not feel the desire of finding exciting and potentially money-attracting names for materials that are the subject of one's present studies? Certainly we also in the bi-anisotropics field have to admit this as we are talking about exotic, novel, or complex materials. But we have to remember that in some cases language can become a barrier for communication.

In the present article the use of the new term, metamaterials, is discussed. I try to find a meaning for it. The term is increasingly being used in literature, and I'll analyse some of the presented definitions. It seems that with the use of the term, 
there is a strong emphasis on the requirement that a metamaterial should display properties that are qualitatively different from its components. My aim is to problematise this requirement, at least in its strong form. I'll focus on the question what is the difference between "ordinary" composite materials and new metamaterials, if any. Towards the end of the article, I shall discuss some examples of complex materials, especially from the point of view of our bianisotropics community, and raise the question to what extent these media deserve to be called metamaterials.

But let us start from definitions.

\section{Metamaterials? What do you mean?}

It is difficult to find an acceptable definition for the term "metamaterial." This statement may sound very normative; after all, can one argue names? Of course, in a free world everyone is allowed to use words in ways that have not necessarily been formally approved into use by international committees, or even to use them in a totally idiosyncratic manner. But to be able to conduct constructive discussion where a given word has an important role, however, it is helpful if at least a group of people agree on the meaning of this word. Sure, the question remains open how large and representative this group must be. Furthermore, with a definition and use of terms in new environments one should pay respect to the historical use of the word, even if its background is on another, perhaps very different, field. This question is not unimportant in the case where we name scientific or engineering things and properties. One example of such a situation is the use of term "metamaterial."

By the writing of the present text (May 2002), the term "metamaterial" has been appearing in print for only a very short period. But some definitions exist. In first-order literature, materials conferences and research web pages the following definitions can be already found:

X Electromagnetic metamaterials are artificially structured composite materials that can be engineered to have desired electromagnetic properties, while having other advantageous material properties. [1]

$x \quad$ Structures composed of macroscopically scattering elements. [2]

$x$ "Meta-materials", materials whose permeability and permittivity derive from their structure. [3]

$x$ MetaMaterials are a new class of ordered nanocomposites that exhibit exceptional properties not readily observed in nature. These properties arise from qualitatively new response functions that are not observed in the constituent materials and result from the inclusion of artificially fabricated, extrinsic, low dimensional inhomogeneities. [4]

X Metamaterials are defined as macroscopic composites having a man-made, three dimensional, periodic cellular architecture designed to produce an 
optimized combination, not available in nature, of two or more responses to a specific excitation. Each cell contains metaparticles, macroscopic constituents designed with low dimensionality that allow each component of the excitation to be isolated and separately maximized. The metamaterial architecture is selected to strategically recombine local quasi-static responses, or to combine or isolate specific non-local responses. [5]

These four attempts to catch the meaning of metamaterials occur with only little variation in the electromagnetics literature. Regarding such definitions, one observation that can be made is that two essential properties are mentioned. The materials should exhibit (electromagnetic) properties

i not observed in the constituent materials, and/or

i not observed in nature.

These are quite remarkable and very restrictive features for materials if they are really applied to define a metamaterial. But indeed, the fact that such properties make a compulsory requirement is in the following sense in consonance with the authentic meaning of the compound word, meta-material.

The prefix meta comes from Greek (it is also used in Latin), and it means "after." Often the prefix is used also for "along with," "beyond," "among," "in the midst of," "according to," and "behind." There are numerous words in English and other languages starting with "meta," like, for example, metabolism, metalinguistics, metamerism, metaphysics, ${ }^{1}$ metamorphosis, metastasis, etc. However, the common word in materials engineering, "metal," is of different origin.

With this background in mind, it seems that in the choice and use of "metamaterial," this meta-prefix emphasises the transcendental aspect of the meaning. A metamaterial needs to be qualitatively different from its components. New phenomena and novel properties should emerge when "ordinary" pieces are brought together. The whole should be more than the sum of its parts. Quantitative differences become qualitative ones.

There is a word for such a behaviour of systems. It is "emergence."

\footnotetext{
${ }^{1}$ The use of "meta" in the term "metaphysics" does not come from a very sublime sense of "above." Rather, it originally applied to those writings of Aristotle which in the accepted order came after (=meta) those dealing with natural science (ta physika--physics, nature) [6]. It is helpful to remember this in today's world where the word metaphysics brings to mind quite obscure, unclear, and transcendental overtones. Even the dictionaric definitions for metaphysics reflect this, being like "the branch of philosophy that treats of first principles, includes ontology and cosmology, and is always intimately connected with an epistemology;" or more generally, "philosophy, esp. in its more abstruse branches" [7].
} 


\section{Emergence. More is different.}

Emergence is the process of emerging. Something arises, comes up into existence. It comes forth from concealment or obscurity. The etymology for this word leads to the combination $e$ (out of) + mergere (to plunge, dip, or immerse). A more common word with the same root is "emergency" which is a sudden, urgent, usually unexpected occurrence or occasion requiring immediate action. But about emergence, it is important to note that it has also a more technical meaning in science and philosophy. In evolutionary theory, it stands for the rise of a system that cannot be predicted or explained from antecedent conditions. George Henry Lewes, the 19th-century English philosopher of science, distinguished between resultants and emergents-phenomena that are predictable from their constituent parts and those that are not [Encyclopaedia Britannica]. Later, emergence theories have been espoused by Henri Bergson, Pierre Teilhard de Chardin, Alfred North Whitehead, and Michael Polanyi. The doctrine of emergence is sometimes stated as part of an "evolutionary cosmogony, according to which the simpler properties and forms of organisation already in existence make contributions to the 'creative advance' of nature by giving birth to to more complex and 'irreducibly novel' traits and structures" [8].

Clearly the present-day electromagnetics use of "metamaterials" is defining the word through the concept of emergence.

The philosophical meaning of "emergence" is connected to another essential term, reductionism. Emergence is very often associated with antireductionistic goals. According to a (strict) reductionist view, "higher-level" scientific theories can be completely and exhaustively reduced to more basic fields of science, for example chemistry into physics, biology in biochemistry, psychology in neurophysiology, and so forth. Everything (it can be objects (ontological reduction), theories, or whole scientific fields) in the higher level of description is contained (even if in a non-obvious way to an amateur) in the "harder" sciences, finally in elementary particle physics. ${ }^{2}$ Clearly the concept of emergence is a problematic phenomenon to a reductionist, or may be difficult to accept within (philosophical) materialism. A person with inclination to a very mechanistically reductionist view may feel the temptation to call an "upper-level" field of science just "applied" more fundamental science: chemistry as applied physics, for example.

In dialectic materialism and Marxism $\square$ Leninism, emergence is nevertheless something that can be accepted. According to those lines of thought, matter (in

${ }^{2}$ It may be visible from the present text that I am not fully subscribing to the strict reductionist programme. Can the great book The Brothers Karamazov by Fyodor M. Dostoyevsky be reduced to (=be equivalent to certain ordered combinations of) the 26 letters of the English alphabet (or, more precisely, to the 33 letters of the Russian one) ?

---I doubt it. (...but feel a little uncomfortable with the doubt...) 
movement) has various levels of existence and movement, physico $\square$ chemical, biological, and social, which all have their own laws. And these laws are independent of the other levels. However, this does not mean that living creatures would possess properties that are not connected to physical laws. It is that they "emerge" in systems that are sufficiently complex but it may be difficult to predict, or even to explain how or when such qualitative changes happen. ${ }^{3}$ A simple extrapolation upwards fails.

Indeed, an important distinction regarding reductionism comes from Philip Anderson [9]. He criticises its strong form which claims that in materials science, there are only "extensive" problems left, equal to device engineering. ${ }^{4}$ Anderson identifies the fallacy in this reasoning in that the reductionist hypothesis does not imply a constructionist one. In other words, even if one could reduce a phenomenon into the fundamental elements, one cannot start with basic laws and reconstruct the universe. "[T]he more the elementary particle physicists tell us about the nature of the fundamental laws, the less relevance they seem to have to the very real problems of the rest of science, much less to those of society."

Emergence can also be appreciated in the mechanism according to which many cellular automata operate. There, simple and local rules can create behaviour in which new patterns may appear and objects are created. Or at least one would think of them as reals "objects" even if they may be just little squares on the computer screen which are turning on and off. For example, in the emergence applets of the Media Laboratory of the Massachusetts Institute of Technology (available from el.www.media.mit.edu/groups/el/projects/emergence) the emergence of "life" can be seen on the computer screen.

\section{Against emergence. More is less.}

But somehow the slogan "the whole is more than the sum of its parts" is counterintuitive. When we look at an ensemble, very much of the detailed structure is left out in the grand picture. If we have a mixture of a great number of elementary objects, in the "real life" they are interacting in many ways. But in the large-scale picture, all these interactions only have an effective influence. The macroscopic picture is a certain "average" of the individual contributions, although this averaging does not need to be a simple operation. And hence extremely many

${ }^{3}$ Something essential about emergence, and about quantity transforming to quality is contained in the following anecdote (taken from the article [9]) where the two famous American literary figures meet in Paris in the 1920's:

FITZGERALD: The rich are different from us.

HEMINGWAY: Yes, they have more money.

${ }^{4} \mathrm{~V}$. Weisskopf [10] distinguishes between intensive and extensive research: "[I]ntensive research goes for the fundamental laws, extensive research goes for the explanation of phenomena in terms of known fundamental laws.... Solid state physics, plasma physics, and perhaps also biology are extensive. High energy physics and a good part of nuclear physics are intensive." 
degrees of freedom are lost when the ensemble is sensed with a coarser resolution. Then, sure, the whole is less than the sum of its parts.

Indeed, an effective picture means a reduced dimensionality compared to the corresponding macroscopic properties of the constituents. One is unable to catch the details because there is simply too much information.

What, then, about the intuitively emergent meaning of a piece of art? Wouldn't everyone take an antireductionist stance when it comes to literature? Why do we feel that a poem is "more" than the information content (expressable as a relatively short sequence of 0 's and 1's) of its letters, exclamation marks and spaces between characters? The answer to this dilemma may be in the following. Is it that when asking such questions we are mixing up the real physical object and the measuring instrument? ${ }^{5}$ What is a poem, after all? And where does its meaning exist?

\section{Physics and metaphysics of electromagnetic materials}

Can the philosophically flavoured discussion in the previous sections be made relevant to electromagnetic properties of materials? Is it too far into the field of metaphysics? Why, the effective modelling of heterogeneous materials is fundamentally analysis and prediction of emergence. When higher-level description of matter is sought using various mixing rules $\square$ even the simplest ones, like Maxwell Garnett [12] $\square$ it quite often happens that the homogenised medium displays properties that are astonishingly different from not only those of the components but also from those that one would expect from the mixing process [13]. But does the fact that the result for macroscopic material character is unforeseen and unexpected automatically mean that the higher-level property is emergent, especially in the evolutional and vitalist tone and meaning of the word? It seems that this is a crucial distinction to be made in the metamaterials discussion.

\subsection{IS A COMPOSITE MATERIAL METAMATERIAL?}

The electromagnetics community has been working for a long time with modelling of properties of heterogeneous materials, both in the field of natural, geophysical materials that are random in structure in the first place, and also in the design of artificial and composite materials where the goal is to manufacture materials with desired properties. In the analysis of the effective properties of such materials, emergence-type behaviour often takes place when we move upwards from the microscale to meso- and macroscales and assign the medium effective properties on the larger scale. But can we call any kind of higher-level material by the name metamaterial?

5 As Kari Enqvist [11] has noted, we may be especially susceptible to this fallacious reasoning when the observing system is the human brain. 
Why not? At least all materials are composites because if we go to smaller and smaller scales, finally we encounter atomic constituents, electrons, protons, neutrons. On macroscale, the medium appears always as an effective average of the lower-level constituents. These effective properties are always "emergent" properties, and are in principle "other than" the properties of the components. They are not plain volume averages of the corresponding inclusion properties, and therefore all matter, even simple mixtures, deserve to be called "metamaterials." And very often do these materials have superior properties over their components. Take for example cellulose or cement, where the superiority is evident even from an everyday-life point of view.

The question is therefore on scale. When we look with myopic eyes at a medium, there is a limit below which we cannot resolve finer details. Can we find or determine an absolute scale? One can always go to higher levels on the scale of various materials. Then we face the problem of the measure and length of homogenisation and the question what can be called a material. Even the mobile radio channel has been called as a complex medium. ${ }^{6}$ What a composite!

\subsection{LINEARITY AND SUPERPOSITION}

Scale and geometry are essential in the character of mixed matter but so is also the constitutive response of the component materials themselves. Nonlinear media are more complex than media with purely linear response. It would be tempting to associate the emergent properties in material description to nonlinearity in the constitutive relations. If the electric polarisation is not linearly dependent on the excitation field, it is certainly difficult to foresee what kind of macroscopic responses can a heterogeneous aggregate of such materials possess. But this kind of a straightforward correspondence between emergence and nonlinearity is not satisfactory, for at least two reasons.

Firstly, the principle of superposition, which according to normal textbook logic follows from linear input--output relation, is still fully compatible with the reductionist view where the properties of the whole can be derived and constructed from the properties of its components. Nonlinearity may lead into difficulties in the analysis of the problem but does not give to it any mystical and unexplainable character. It is only that we can no longer exploit superposition and splitting of the problem into easily solvable subproblems.

For the second, even simple, linear mixing problems may bring forth macroscopic phenomena that quite often look emergent in the unexpected sense of the word. Such phenomena, like for example, percolation, enhanced electric or magnetic polarisation, and chirality, will be discussed later in this section.

\footnotetext{
${ }^{6}$ See the title of the presentation by R. Prasad and D.K. Kalluri in the Conference Complex Mediums II: Beyond Linear Dielectrics, in SPIE's $46^{\text {th }}$ Annual Meeting and International Symposium on Optical Sciences and Technology, 4467, San Diego, California, 2001.
} 
Our eyes as receiving antennas and the image processing equipment connected to those make their own interpretations of the random microstructure of materials. Take, for example, the two-dimensional "material" depicted in Figure 1. There, depending on scale, structures may be seen as emergent patterns, although the dew formation on a day with freezing environment follows blind rules of condensation.

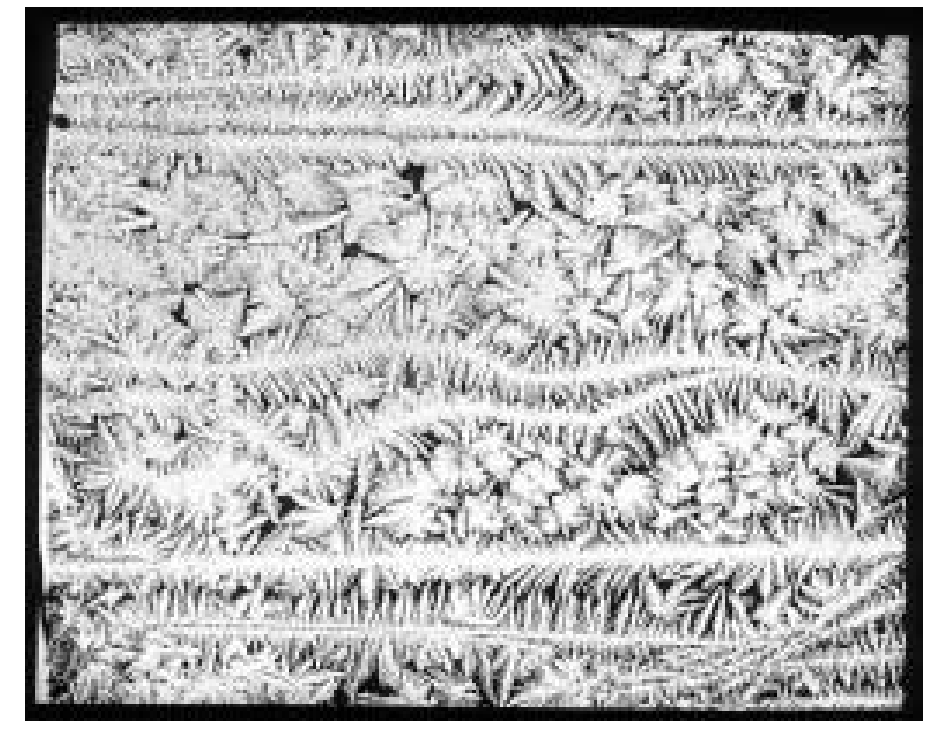

Figure 1. Frost and dew form patterns in which structure on a higher level clearly emerges. (Perhaps emerges ...) This picture is from W.A. Bentley's snow archives [14].

And how blind is the process? The rules and laws governing the evolution and rest states of the physical system may be written as partial differential equations as has been the habit throughout the three centuries old history of mathematical physics. No emergence is explicit in these equations. They only connect the amplitudes at a certain spatial point at a certain time instant to the neighbouring ones. Perhaps the alternative description in which the observed phenomena of the material world are seen as products of rules, simple lines of code which Stephen Wolfram [15] has advocated may catch more of the essence of these emergent phenomena.

\subsection{ARTIFICIAL DIELECTRICS}

An earlier version of the metamaterial-term is the term "artificial dielectric" which has a history of over a half century. In 1948 Winston E. Kock suggested to make a dielectric lens lighter in weight by replacing the refractive material by a mixture of metal spheres in a matrix. Kock built lenses by spraying conducting paint on 
polystyrene foam and cellophane sheets. He was probably the one to coin the term artificial dielectric, the physics of which is well documented in Collin's text on guided waves [16] and which term is very well established in the microwave literature (see also, [17]). Let us remind ourselves of Kock's own words [18]:

The artificial dielectric material which constitutes the delay lens was arrived at by reproducing, on a much larger scale, those processes occurring in the molecules of a true dielectric which produce the observed delay of electromagnetic waves in such dielectrics. This involved arranging metallic elements in a three-dimensional array or lattice structure to simulate the crystalline lattices of the dielectric material. Such an array responds to radio waves just as a molecular lattice responds to light waves; the free electrons in the metal elements flow back and forth under the action of the alternating electric field, causing the elements to become oscillating dipoles similar to the oscillating molecular dipoles of the dielectric.

Compare this with the introductions of reports from the present third millennium that deal with photonic band gap structures and metamaterials! Although in the above citation the emphasis of low frequencies is conspicious and therefore does not directly relate to more complicated resonance phenomena, this excerpt is a good reminder for us about the fact that many ideas in materials design have been around for a long time.

Admittedly today's treatments of the metamaterial design are more detailed. Take, for example, the classification into mesomaterials and metamaterials by Walser [5]. With mesomaterial design he means that ordered macroscopic materials are synthesised from the bottom up by assembling macromolecules, like fullerenes or carbon nanotubes (these are so-called mesoscale supramolecules). In the more advanced and mature metamaterials design the order is the opposite: macroscopic composites are synthesised top down by "first disassembling, then recombining macroscopic constituents." Probably the strategy in this second approach by Walser is more target-oriented: the desired properties of the metamaterial to be fabricated are predetermined with stricter tolerances as in the first, mesomaterial approach.

\subsection{EMERGENCE AND REDUCTION IN ELECTROMAGNETICS: ARE THEY COMPATIBLE?}

The crucial question remains: does there exist really emergent behaviour when electromagnetic materials are treated as mixtures and when they are synthesised from lower-level components? An emphatic yes is clearly the opinion of many metamaterials investigators. Or, looking from another direction, is everything in the behaviour of the composite on a higher level just a straightforward average of the properties of its components and the microgeometry of its structure, which would be a more reductionistic view? To make it easier to look for answers for these questions, Table 1 attempts to achieve structure to the dichotomy between 
emergence and reduction in the degrees of freedom in the description of electromagnetic materials.

TABLE 1. Some differences follow regarding the status of "ordinary" composites, when the point of view of emphasising emergence is changed to a reductionistic one. The left column (the whole is MORE than the sum of its parts) stresses the view of qualitatively different character of engineered metamaterials with emergent properties, while the right-hand side (the whole is LESS than the sum of its parts) takes the more mechanistic bottom-up view of material description where the averaging process reduces the dimensionality of the microlevel of the matter.

\begin{tabular}{|c|c|c|}
\hline $\begin{array}{l}\text { The whole vs. } \\
\text { the sum of its } \\
\text { parts }\end{array}$ & MORE & LESS \\
\hline $\begin{array}{l}\text { Philosophical } \\
\text { background } \\
\end{array}$ & $\begin{array}{c}\text { Idealistic, immaterialistic, } \\
\text { holistic } \\
\end{array}$ & $\begin{array}{c}\text { Reductionist, } \\
\text { physicalist }\end{array}$ \\
\hline $\begin{array}{l}\text { Effect on the } \\
\text { higher level of } \\
\text { description } \\
\end{array}$ & Emergence & $\begin{array}{c}\text { Reduced } \\
\text { dimensionality } \\
\end{array}$ \\
\hline Materials & Metamaterials & Classical composites \\
\hline $\begin{array}{l}\text { Effective medium } \\
\text { description }\end{array}$ & $\begin{array}{c}\text { Non-local effects and } \\
\text { connections }\end{array}$ & Linear mixing rules \\
\hline $\begin{array}{l}\text { Effects and } \\
\text { phenomena }\end{array}$ & Spontaneous symmetry breaking & $\begin{array}{c}\text { Continuous } \\
\text { extrapolation }\end{array}$ \\
\hline Examples & $\begin{array}{l}\text { Percolation, chiral materials, } \\
\text { artificial (and natural) } \\
\text { magnetism, PBG's, Veselago } \\
\text { media }\end{array}$ & $\begin{array}{c}\text { Simple aggregates, } \\
\text { laminates, weakly } \\
\text { interacting inclusions }\end{array}$ \\
\hline
\end{tabular}

In the table the contrast between the two views appears perhaps a little exaggerated but it is intentional: to make the translation of the philosophical thinking to the electromagnetics level. Emergence is identified with new macroscopic phenomena that are brought forth by the process of mixing. But it is at the same time contrasted with the recipes of classical mixing formulas which may look quite simple algebraic relations between the properties of the composite on one hand, and those of the constituent materials on the other. But such a picture may need more elaboration. Let us take a closer look at various materials in electromagnetics and see how they relate to the dichotomy of Table 1 .

\subsection{EXAMPLES OF ELECTROMAGNETIC MATERIALS WITH EMERGENT PROPERTIES}




\subsubsection{Chiral materials}

From the point of view of complex materials and bianisotropic description of electromagnetic materials, the obvious example of media with intuitively emergent properties is the class of chiral materials. In chiral media, the geometrical character of the internal structure (the handedness) cause macroscopic effects that are observed as the rotation of the polarisation of the propagating field plane [19]. A man-made chiral sample could be a collection of metal helices, randomly oriented and distributed in a neutral polymer matrix. Were one to call chiral media metamaterials with emergent properties, the emergent character could be interpreted in the manner that nothing in the component materials (metal and polymer) hints to the ability of causing rotatory power but still this is the effect of the chiral composite. The effect comes through mixing and geometry.

\subsubsection{Percolation effects}

Percolation effects display themselves as large changes in macroscopic properties of random materials when the fractional volumes of the components are varied [20]. Percolation is an important phenomenon and it has been studied in connection with several fields and applications: study of ferromagnetism, soil moisture, oil penetration in rocks, the spread of epidemics, forest fires, and even in wafer-scale integration in the manufacture of microchips. Also the macroscopic electromagnetic properties of random materials can achieve percolatory character, being very sensitive to the microstructure of the mixture, even if the components have no special character in their properties.

\subsubsection{Artificial magnetics}

One further example of the metamaterial-type phenomenon is the creation of magnetism without magnetic constituents. This may happen in ordinary random materials, like wet snow where loop-forming parts of the liquid water cause diamagnetic behaviour in the macroscopic response of the medium. The magnetic permeability, $\mathrm{P}$ is not equal to that of the free space. But this effect can also be enhanced and "artificially" produced by embedding various metallic loop-type elements in a matrix. Swiss roll -type of scatterers are especially effective in this respect [21]. Also in modern metamaterial design the use of split-ring resonators (which often are adjacent, nonconnected open metallic loops) is quite common. Because of its planar structure it is perhaps a more practical way of creating artificial magnetism [22].

\subsubsection{Enhanced polarisation}

The next example of "emergent behaviour" due to simple mixing is so-called enhanced polarisation. This phenomenon is even theoretically visible with the use of the most simple mixing rule, the Maxwell Garnett formula for mixtures where spherical scatterers, small compared to the wavelength, are immersed in 
homogeneous background. Figure 2 displays the results of such mixing in two cases.

The two figures illustrate clearly the two classes of mixtures in Table 1. On the left, the macroscopic permittivity of dry snow is a quite dull and uninteresting average of the permittivities of its two constituents, air and ice. Nothing emergent--qualitatively new---can be observed. But on the right-hand figure, the real part of the effective permittivity of the mixture where lossy spheres are embedded in air displays much stronger polarisation (the real part of the effective permittivity is close to 5 even if the real part of the inclusions is 2) than either of the constituents. This happens, of course, only at a range of the volume fraction of the inclusions (here the maximum enhancement requires around $80 \%$ fraction). And this enhancement can be made much stronger by increasing losses in the inclusion phase. This effect of high permittivity values is known with living matter, where interfacial polarisation mechanisms may be drastically enhanced and observed with biological membranes and tissues.


Figure 2. The (real part of the) effective permittivity of a mixture with spherical dielectric inclusions immersed in free space, as a function of the volume fraction of the inclusions. Left-hand side: dry snow (the inclusions are ice (permittivity $¥ 3.17$ ) and background is air (permittivity $¥ 1$ )). Right-hand side: a high-loss mixture with inclusions having permittivity $¥ 2$ - j10 in air. (Note that depicted is the real part of the permittivity.)

\subsubsection{Veselago medium}

The so-called Veselago medium can be deservedly called a metamaterial par excellence. This medium has, as very often new effects and inventions do, several different names:

i Left-handed medium

i Medium with simultaneously negative permittivity and negative permeability

i Negative index of refraction (NIR) medium

i Double negative metamaterial 


\section{i Backward-wave material}

Suggested in 1967 [23], this medium has been studied theoretically and experimentally very much during the two last years or so. As can be inferred from the various labels on Veselago medium, its nonconventional property is that both its permittivity and permeability are negative, for a certain range of frequencies. Practical realisations have been indeed achieved for such Veselago-medium samples with observed properties that confirm the predictions (see, for example, [24]). The strange effects of Veselago media include anomalous refraction, inverted Cherenkov radiation and Doppler shift, and it is being studied for applications involving waveguiding, radiation, and diffraction phenomena.

In many of these emergent phenomena that are created in mixtures, the effect is a cause of a break in the symmetry that is preserved on the lower level. No wonder that broken symmetry is an important concept in the description of magnetism. But even in more general terms, many material effects can be said to testify to Johannes Kepler's famous slogan (see, for example, [25]): Ubi materia, ibi geometria.

\section{Conclusion: the three critiques}

To conclude this treatment of emergence in electromagnetic materials, let us summarise the critique towards the definition of metamaterial when it is understood in the above sense that has been deconstructed from the explicit definitions and practical use of the term.

Firstly, the requirement that a metamaterial needs to be "superior" to previously existing materials is inherently subjective. There are figures of merit with which people measure superiority of the materials. For example, in ferrites this measure could be the product of low-frequency permeability and resonant frequency. But because of this arbitrariness, a definition based on superiority is too loose to be acceptable to classify metamaterial. Any kind of new mixture is always different from previously existing ones and therefore possesses a unique combination of properties. If such a set is what is wanted, we have everywhere examples of metamaterials.

The second piece of critique is connected to the distinction between local and non-local effects in the material response, also mentioned in Table 1. Very often the emergent and novel property of metamaterials is assigned to the fact that the scattering elements in the composite interact in a non-local manner, like for example in frequency-selective surfaces or photonic crystals. Spatial dispersion is often required for the higher-order effects. But this is not necessary, as has been pointed out in the previous examples in this article. Even simple mixing rules are able to explain very non-intuitive and emergent properties for ordinary-looking mixtures, like, for example, enhanced polarisation and percolation behaviour.

And finally, if a metamaterial should possess properties that (in addition of being superior to those observed before) are not found in nature, the definition might sound a little contradictory. We are, in our design and synthesis of composite 
materials, working according to the rules and laws of nature. ${ }^{7}$ Therefore the results $\square$ our metamaterials $\square$ also are "natural." And if we did not find materials with properties corresponding to these new designs yet in nature, is it only that we did not search hard enough? Nature is extremely prodigious. All possibilities come true. All properties, all media that are not forbidden, are there, somewhere. They are compulsory.

\section{References}

[1] The www page of David Smith, University of California, San Diego. http://physics.ucsd.edu/ drs/

[2] Weiland, T., Schuhmann, R., Greegor, R.P., Parazzoli, C.G., Vetter, A.M., Smith, D.R., Vier, D.C., and Schultz, S. (2001) Ab initio numerical simulation of left-handed metamaterials: Comparison of calculations and experiments, $J$. of Applied Physics, 90(10), 5419-5424.

[3] Pendry, J.B. (2001), Negative $P$, negative $H$ negative refractive index, and how to exploit them. Electromagnetic Crystal Structures, Euroconference on Electromagnetic Confinement, from Basic Research to the Marketplace, St. Andrews, Scotland, 9-14 June 2001. (no page number)

[4] Metamaterials home page of the future projects of the Defense Advanced Research Projects Agency's (DARPA) Defense Sciences Office (DSO). http://www.darpa.mil/DSO/future/metamaterials/metamaterials.html

[5] Walser, R.M. (2001) Electromagnetic metamaterials, Inaugural Lecture, Proc. of SPIE (Complex Mediums II: Beyond Linear Isotropic Dielectrics; Lakhtakia, A, Weiglhofer, W.S., and Hodgkinson, I.J.editors), 4467, 1--15.

[6] Chambers Twentieth Century Dictionary, 1977, (edited by A M Macdonald)

[7] The Random House Dictionary of the English Language, Second Edition, unabridged, New York, 1987, (Stuart Berg Flexner, editor-in-chief).

[ The "abstruseness" of the Random House definition for metaphysics is evident in the following citation from "Subjects among other things" by E. Sosa (1987) in Philosophical Perspectives, Vol. 1, where also "metamaterial" is used in a different meaning (adjective) as in the present article: "If we allow real status to the materially derivative, it seems arbitrary to rule out objects that though immaterial are no more derivative; all the more so if in each case the mode of derivation is equally well

7 The question of what kind of materials can occur in nature and which are forbidden may bring reminiscences into mind of the controversy concerning the possible existence of nonreciprocal bi-isotropic media, also called as NRBI media or Tellegen media [26], [27]. However, there the discussion focused on the question whether the laws of physics forbid the possibility to manufacture these materials (in which case it would be understandable that one cannot find those in nature). Here, with metamaterials discussion, no one is claiming that metamaterials as such break the rules acconrding to which materials have to exist; it is only that some metamaterials definitions exclude the natural existence of such media, even if engineers can (and want to) manufacture those. 
understood. So it seems ill-advised and unnecessary to strain against the immaterial, or at least against that which is sufficiently meta-material (metaphysical?) to be distinct from the chunk of material that constitutes it at that time." (italics not in the original) ]

[8] Nagel, E. (1979) The structure of science, Problems in the logic of scientific explanation.Routledge and Kegan Paul, New York.

[9] Anderson, P.W. (1972) More is different. Broken symmetry and the nature of the hierarchical structure of science. Science, 177(4047), 393-396.

[10] Weisskopf, V.F., in Brookhaven Nat. Lab. Publ. 888T360. Cited in [9].

[11] Enqvist, K. (2000) Valo ja varjo (Light and shadow, in Finnish). WSOY.

[12] Maxwell Garnett, J.C. (1904) Colours in metal glasses and metal films, Transactions of the Royal Society (London), CCIII, 385-420.

[13] Sihvola, A. (1999) Electromagnetic mixing formulas and applications, IEE Publishing, Electromagnetic Waves Series, London.

[14] Wilson "Snowflake" Bentley Digital Archives. The man \& his images, CDrom, 1, PhotoGraphics Multimedia production, Jericho, Vermont, USA, 1999. See also, Bentley, W.A. and Humpreys, W.J. (1962) Snow Crystals. New York: Dover.

[15] Wolfram, S. (2002) A new kind of science, Wolfram Media, Inc.

[16] Collin, R.E. (1991) Field theory of guided waves, Second Edition, IEEE Press, New York.

[17] Brown, J. (1960) Artificial dielectrics, in Progress in Dielectrics, (Birks, J.B., editor) 2, 193-225.

[18] Kock, W.E. (1948) Metallic delay lenses, Bell System Technical J., 27, 58-82.

[19] Lindell, I.V., Sihvola, A.H., Tretyakov, S.A., and Viitanen, A.J. (1994) Electromagnetic waves in chiral and bi-isotropic media, Artech House, Boston and London.

[20] Grimmett, G. (1989) Percolation, Springer, New York.

[21] Pendry, J.B., Holden, A.J., Robbins, D.J., and Stewart, W.J. (1999) Magnetism from conductors and enhanced nonlinear phenomena, IEEE Transactions on Microwave Theory and Techniques, 47(11), 2075-2084.

[22] Shelby, R.A., Smith, D.R., and Schultz, S. (2001) Experimental verification of a negative index of refraction, Science, 292, 77-79.

[23] Veselago, V.G. (1968) The electrodynamics of substances with simultaneously negative values of $\mathrm{H}$ and $\mathrm{P}$, Soviet Physics Uspekhi, 10(4), 509-514. (Translation from the original Russian version, Uspekhi Fizicheskii Nauk, 92, 517-526, July 1967.)

[24] Shelby, R.A., Smith, D.R., Nemat-Nasser, S.C., and Schultz S. (2001), Microwave transmission through a two-dimensional, isotropic, left-handed metamaterial, Applied Physics Letters, 78(4), 489-491.

[25] Sihvola, A. (2000) Ubi materia, ibi geometria. Helsinki University of Technology, Electromagnetics Laboratory Rep. Series, 339, September 2000. See also http://www.hut.fi/ asihvola/memos.html 
[26] Lakhtakia, A. and Weiglhofer, W.S. (1994) Are linear, nonreciprocal, biisotropic media forbidden? IEEE Trans. Microwave Theory and Techniques, 42(9), 1715-1716. (See also, ibid., September 1995, December 1995)

[27] Sihvola, A.H. (1995) Are nonreciprocal, bi-isotropic media forbidden indeed? IEEE Trans. Microwave Theory and Techniques, 43(9), 2160-2162. (See also, ibid., September 1994, December 1995) 